\title{
Spectral Filtering of the Spatial Green's Function
}

\author{
F. Vipiana*(1) A. Polemi ${ }^{(2)}$, S. Maci ${ }^{(3)}$, and G. Vecchi ${ }^{(1)}$ \\ (1) LACE, Politecnico di Torino, C.so Duca degli Abruzzi 24, 10129 Torino, \\ Italy, francesca.vipiana@polito.it, giuseppe.vecchi@polito.it \\ (2) Dep. of Information Engineering, Università di Modena e Reggio Emilia, Via \\ Vignolese 905, 41100 Modena, polemi.alessia@unimore.it \\ (3) Dep. of Information Engineering, University of Siena, Via Roma 56, 53100 \\ Siena, Italy,macis@ing.unisi.it
}

\section{Introduction}

In the Method-of-Moments (MoM), applied to the Electric Field Integral Equation (EFIE), the solution (the unknown current) is approximated by a linear combination of a finite number of basis functions. The basic question addressed in this work is whether the use of the exact Green's function is the optimal choice once the (finite-dimensional) space of the approximate solution has been chosen. We approach to the above question introducing a new representation of the spatial Green's function obtained by an appropriate spectral filtering with a meshdependent spectral content. The first direct impact of this filtering is in the disappearance of the Green's function singularity; as a consequence, the spatial integration is easier, avoiding the extraction of the singular term in the integration process.

Here we consider both scattering and antenna problems in free space, proposing a "band limited" Green's function expressed in closed form in the spatial domain and obtained through an appropriate windowing in the spectral domain.

In [1] preliminary investigations have been done for periodic structures. For 2D scattering problems [2] proposed a spectrally-filtered Green's function that has to be obtained numerically; for 3D scattering problems [3] proposed a regulated kernel, obtained with a simple windowing function, evaluated through a series.

\section{Formulation}

Using the standard spatial and spectral version of the MoM (Parseval theorem), the $(m, n)$-entry of the MoM matrix is expressed as:

$$
Z_{m n}=\iint_{\Sigma_{m}} d^{2} \underline{\rho} \underline{f}_{m}(\underline{\rho}) \cdot \iint_{\Sigma_{n}} d^{2} \underline{\rho^{\prime}} \underline{\underline{G}}\left(\underline{\rho}-\underline{\rho}^{\prime}\right) \cdot \underline{f}_{n}\left(\underline{\rho}^{\prime}\right)=\frac{1}{4 \pi^{2}} \iint_{\mathfrak{M}^{2}} d^{2} \underline{\sigma} \underline{\tilde{f}}_{m}(-\underline{\sigma}) \cdot \underline{\underline{G}}(\underline{\sigma}) \cdot \underline{\tilde{f}}_{n}(\underline{\sigma})
$$

where " $\sim$ " indicates Fourier transforms. We analyze the effect of truncating the spectral integration region up to a finite spectral bandwidth $K$, that is equivalent in the spatial-domain to the use of a windowed band limited Green's function $G^{W}$ : 


$$
Z_{m n}(K)=\iint_{\Sigma_{m}} d^{2} \underline{\rho} \underline{f}_{m}(\underline{\rho}) \cdot \iint_{\Sigma_{n}} d^{2} \underline{\rho}^{\prime} \underline{\underline{G}}^{W}\left(\underline{\rho}-\underline{\rho}^{\prime}\right) \cdot \underline{f}_{n}\left(\underline{\rho}^{\prime}\right)
$$

where $\underline{\underline{G}}^{W}(\underline{\rho})=\frac{1}{4 \pi^{2}} \int_{-K}^{K} \int_{-K}^{K} d \sigma_{x} d \sigma_{y} \underline{\underline{\widetilde{G}}}(\underline{\sigma}) \cdot e^{-j \underline{\sigma} \underline{\underline{\rho}}}$.

The criterion to find the optimal spectral threshold $K$ is related to the main lobe of the employed basis functions spectra. This is related to the reciprocal of the mesh size $\Delta$ (the relation is explicit and trivial for separable functions like rooftops), obtaining $K \propto 2 \pi / \Delta$. The simplest filtering is a rectangular window that drops abruptly to zero at $K$, but this truncation lends to produce ripples in the space domain, and related Gilbbs phenomena; so in the following we use more convenient spectral windows [4].

\section{Band-limited regularized free space Green's function}

In free space, the "infinite-bandwidth" (i.e. exact) Green's function is known in closed form, so we would like to maintain this property in its band-limited version. From the Sommerfeld identity representation of the Green's function,

$$
g\left(\rho, z, z^{\prime}\right)=\frac{e^{-j k_{0} r}}{r}=\frac{1}{2 \pi} \int_{0}^{\infty} \frac{e^{-j k_{z}\left|z-z^{\prime}\right|}}{j k_{z}} J_{0}(\sigma \rho) \sigma d \sigma
$$

we can directly derive the band-limited free space Green's function $g^{w}$ :

$$
g^{W}\left(\rho, z, z^{\prime}\right)=\frac{1}{2 \pi} \int_{0}^{\infty} w(\sigma) \frac{e^{-j k_{z}\left|z-z^{\prime}\right|}}{j k_{z}} J_{0}(\sigma \rho) \sigma d \sigma
$$

where $r=\sqrt{\rho^{2}+\left|z-z^{\prime}\right|^{2}}, k_{z}=\sqrt{k_{0}^{2}-\sigma^{2}}$, and $w(\sigma)$ is the chosen window.

In order to use a window such that the integral (4) can be evaluated in a closed form, thus avoiding any numerical integration process, we propose first to apply the exponential window $w(\sigma)=e^{-j \alpha k_{z}}$, where $\alpha$ is a parameter related to the mesh size. With this window the integral (4) is evaluated analytically, obtaining from (4) a band-limited Green's function $g^{W}\left(\rho, z, z^{\prime}\right)=\frac{e^{-j k_{0} \tilde{r}}}{\widetilde{r}}$ where $\widetilde{r}=\sqrt{\rho^{2}+\left(\left|z-z^{\prime}\right|+\alpha\right)^{2}}$. To be able to use other kinds of spectral windows, still having a closed form for the windowed free space Green's function, we suggest to expand the chosen window in $M$ complex sources $w(\sigma)=\sum_{i=1}^{M} a_{i} e^{-k_{z} e_{i}}$, obtaining a band-limited Green's function $\quad g^{W}\left(\rho, z, z^{\prime}\right)=\sum_{i=1}^{M} a_{i} \frac{e^{-j k_{0} \widetilde{r}_{i}}}{\widetilde{r}_{i}} \quad$ where $\widetilde{r}_{i}=\sqrt{\rho^{2}+\left(\left|z-z^{\prime}\right|-j e_{i}\right)^{2}}$. The coefficients $a_{i}$ and $e_{i}$ are calculated through the Generalized Pencil of Function (GPOF) procedure [5]. 


\section{Numerical results}

First of all we analyze the scattering from a square flat plate, with a side of $15 \mathrm{~cm}$, discretized by 280 Rao-Wilton-Glisson (RWG) basis functions [6]. The Green's function is filtered with two exponential windows, shown in Figure 1, with bandwidth related do the mesh size $\Delta(-3 \mathrm{~dB}$ bandwidth equal to $4 \pi / \Delta$ and $6 \pi / \Delta)$. The $-3 \mathrm{~dB}$ circles of the considered spectral windows are reported on the amplitude Fourier Transform of the "normal" RWG (constructed as the vector product of the two polar components) in Figure 2.

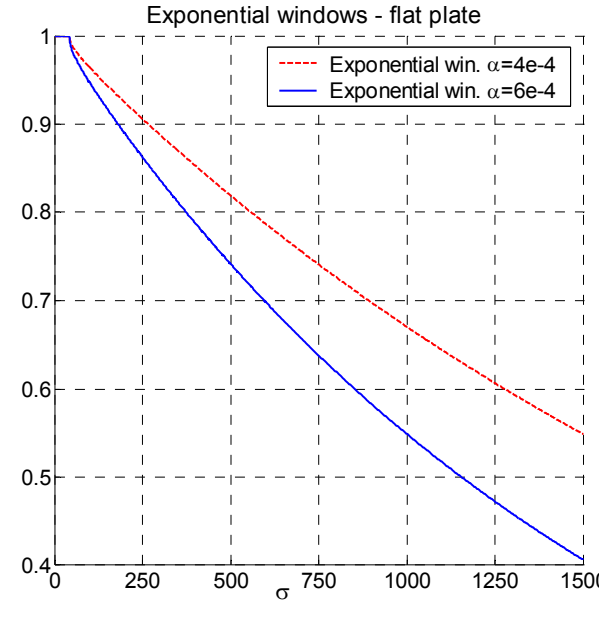

Figure 1: Scattering from a flat plate: exponential window filtering

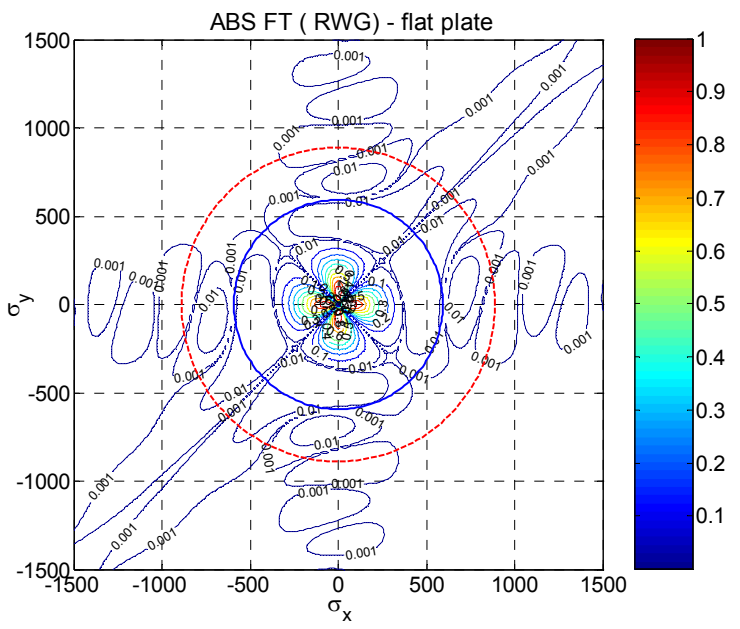

Figure 2: flat plate, FT of a RWG with the $-3 \mathrm{~dB}$ circles of the exponential windows

In Figure 3 the amplitude of the scattered field is shown comparing the case without windowing (exact Green's function) and applying the two exponential windows of Figure 1: the results are overlapped.
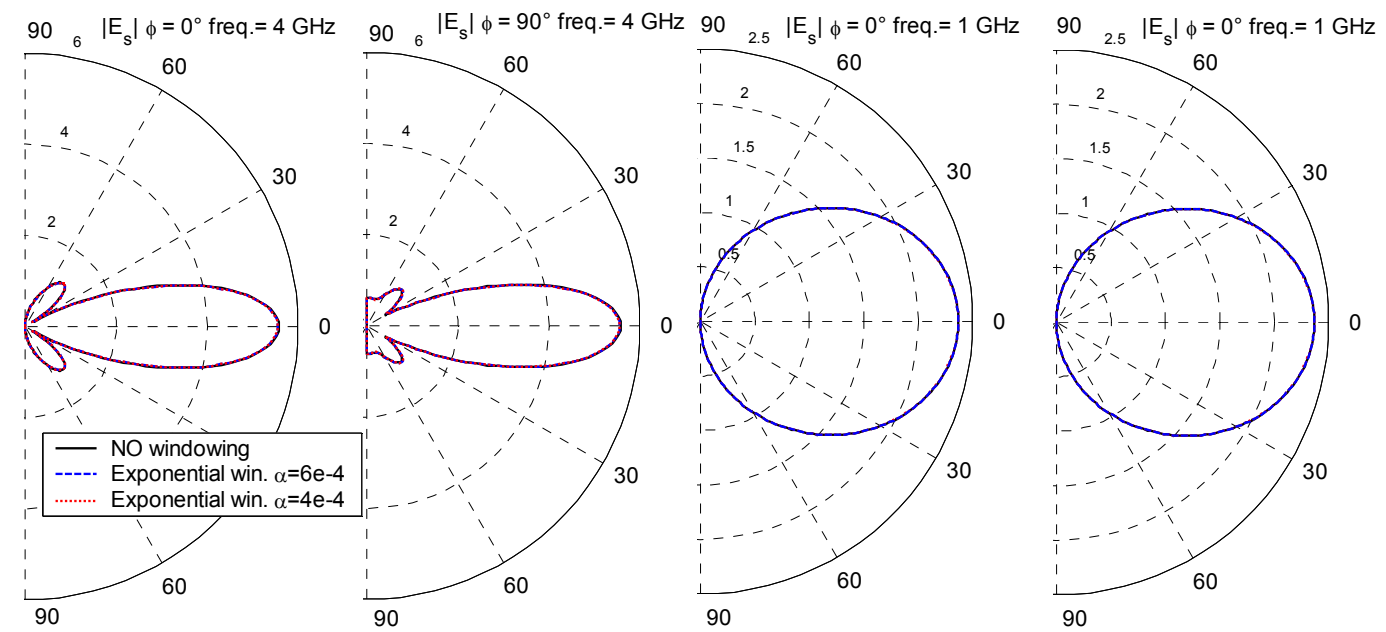

Figure 3: Scattering from a flat plate. Amplitude of the scattered field: comparison between no windowing (solid line) and exponential windowing (dash and dotted lines)

Then we analyze a dipole with length of $75 \mathrm{~mm}$ excited with a voltage gap in the center, in order to evaluate its input impedance. First we apply two exponential 
windows: the filtered Green's function is shown in Figure 4 in dash and dashdotted lines. Figure 5 reports the evaluated input impedance. We can see that, with the exponential windows, the results are not overlapped with the reference obtained with the exact Green's function. So we try another (more convenient) spectral window, a gaussian window [4] expressed as sum of $M=5$ complex sources. The resulting band-limited Green's function is depicted through a solid line in Figure 4 (overlapped with the dotted line obtained without the complex sources expansion), and the evaluated input impedance is very close to the reference obtained without filtering (see Figure 5).

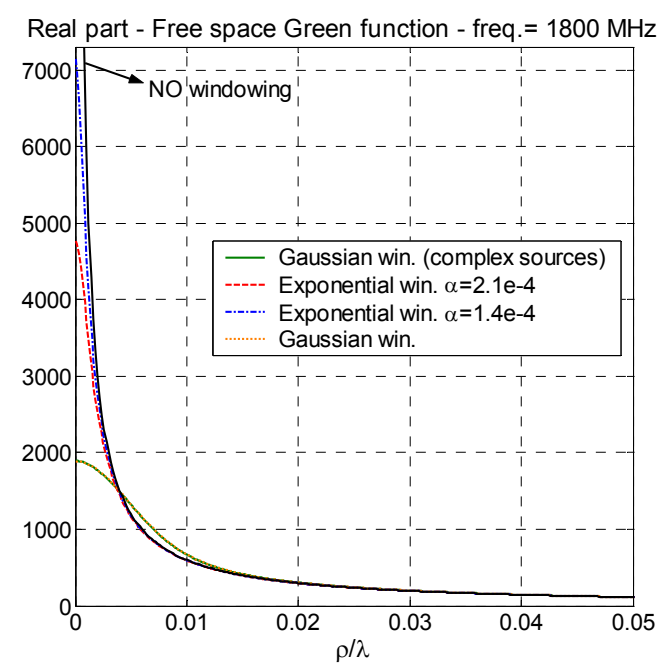

Figure 4: dipole, real part of the free space Green's function, comparison between no windowing, exponential and gaussian windows.

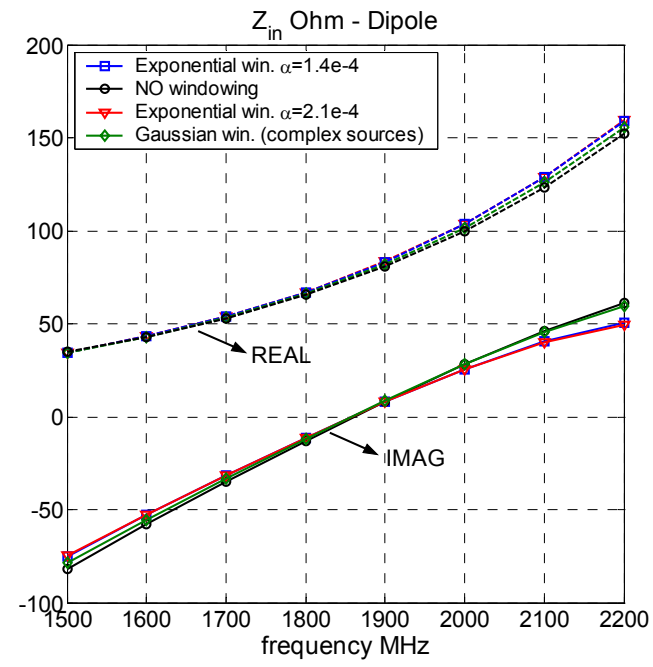

Figure 5: dipole, input impedance, comparison between no windowing, exponential and gaussian windows.

\section{References}

[1] F. Vipiana, A. Polemi, G. Vecchi, S. Maci, "Hybrid Spatial-Spectral Analysis of Periodic Structures", Proc. IEEE Int. Sym. on Ant. Propag., Albuquerque (New Mexico), July 2006, pp. 2867-2870.

[2] G.F. Herrmann, S.M. Strain, "Sampling Method Using Prefiltered BandLimited Green's Functions for the Solution of Electromagnetic Integral Equations", IEEE Trans. Ant. Prop., Vol. 41, No. 1, 1993, pp. 20-24.

[3] K.F. Warnick, G. Kang, W.C. Chew, "Regulated Kernel for the Electric Field Integral Equation", Proc. IEEE Int. Sym. on Ant. Propag., Vol. 4, July 2000, pp. 2310-2313.

[4] F.J. Harris, "On the use of windows for harmonic analysis with the Discrete Fourier Transform", Proc. of the IEEE, vol. 66, no. 1, Jan. 1978.

[5] T. K. Sarkar, O. Pereira, "Using the Matrix Pencil Method to Estimate the Parameters of a Sum of Complex Exponential", IEEE Trans. on Ant. Prop., vol. 37, no.1, pp.48-55, Feb. 1995.

[6] S. M. Rao, D. R. Wilton, A. W. Glisson, "Electromagnetic scattering by surfaces of arbitrary shape", IEEE Trans. Ant. Prop., Vol. 30, pp. 409-418, May 1982. 